\title{
Association between SYNTAX II Score and late saphenous vein graft failure in patients undergoing isolated coronary artery bypass graft surgery
}

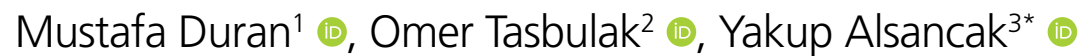

\section{SUMMARY}

OBJECTIVE: Coronary artery bypass graft (CABG) surgery is a well-established treatment modality for patients with multivessel coronary artery disease (CAD). Syntax II Score has been established as novel scoring system with better prediction of postprocedural outcomes. This study aimed to investigate the prognostic value of SYNTAX II Score for predicting late saphenous vein graft (SVG) failure in patients undergoing isolated $C A B G$.

METHODS: The records of 1,875 consecutive patients who underwent isolated CABG with at least one SVG were investigated. Those who underwent coronary angiography and SVGs angiography at least 1 year after the CABG were included. Patients were divided into two groups based on the presence or absence of SVG failure. For each group, predictors of late SVG failure and subsequent clinical outcomes were analyzed.

RESULTS: According to this study, the presence of hypertension, higher rates of repeat revascularization, and higher SYNTAX II Scores were found to be independent predictors of late SVG failure. In addition, the prognostic value of SYNTAX II Score was found to be significantly higher than anatomical SYNTAX Score in terms of predicting late SVG failure and major adverse cardiovascular and cerebrovascular event. CONCLUSIONS: There was a strong association between SYNTAX II Score and late SVG failure in patients undergoing isolated CABG. KEYWORDS: Late saphenous vein graft failure. SYNTAX II Score.

\section{INTRODUCTION}

Saphenous vein grafts (SVGs) are widely used venous conduits for coronary artery bypass graft (CABG) surgery. However, long-term surgical success and subsequent clinical benefits following CABG largely depend on graft patency ${ }^{1-4}$. The rates of SVG failure during the first 12 to 18 months following CABG have been reported to reach approximately $25 \%$ and the patency of grafts progressively decreased following years ${ }^{5,6}$. Due to adverse cardiac events associated with SVG failure, preventing graft failure is of utmost importance ${ }^{7}$.

SYNTAX scoring system was established with the intent of determining whether the percutaneous coronary intervention (PCI) or CABG was preferable in patients with coronary artery disease $(\mathrm{CAD})$ requiring revascularization ${ }^{8}$. Thereafter, SYNTAX II scoring system has been established by integrating anatomical features and clinical characteristics of patients with the intent of achieving better prediction of postprocedural outcomes?

\footnotetext{
${ }^{1}$ Konya Training and Research Hospital, Department of Cardiology - Konya, Türkiye.

${ }^{2}$ Istanbul Mehmet Akif Ersoy Thoracic and Cardiovascular Surgery Training and Research Hospital, Department of Cardiology - İstanbul, Türkiye.

${ }^{3}$ Necmettin Erbakan University Meram Faculty of Medicine, Department of Cardiology - Konya, Türkiye.

*Corresponding author: dryakupalsancak@gmail.com

Conflicts of interest: the authors declare there is no conflicts of interest. Funding: none.

Received on June 22, 2021. Accepted on July 18, 2021.
} 
Although SYNTAX II provides a more accurate and individualized estimate of postprocedural outcomes, its predictive value for the occurrence of late SVG failure is inconclusive.

In this study, we aimed to investigate the prognostic value of SYNTAX II Score for predicting late SVG failure and its association with subsequent clinical outcomes in patients undergoing isolated CABG.

\section{METHODS}

\section{Study design}

In this retrospective study, subjects were selected from the 1,875 patients with multivessel CAD who underwent isolated CABG with at least one SVG between 2009 and 2011. Those who later underwent subsequent coronary angiography and SVG angiography between January 2010 and January 2020 due to stable ischemic findings detected by noninvasive imaging modalities including positive cardiovascular exercise stress testing or myocardial perfusion defects were included in the study. Demographic and clinical characteristics of patients and the indication for the procedure were retrospectively analyzed. Patients who underwent coronary angiography and SVG angiography at least 1 year after the CABG were included and those who did not meet this criterion were excluded. In addition, patients undergoing emergent CABG or concomitant valve surgery, patients with a prior history of severe valvular disease, congenital heart disease, severe organ dysfunction including liver or kidney failure, malignancy, lack of regular follow-up, and those nonadherent to their medical treatment following surgery were excluded. After exclusion criteria had been applied, a total of 280 patients were enrolled in the present study (Figure 1). According to our study, patients were divided into two groups based on the presence or absence of late SVG failure. The obtained data pool was statistically analyzed. Informed consent was obtained from all patients in accordance with a protocol approved by the Ethics Committee of Istanbul Mehmet Akif Ersoy Thoracic and Cardiovascular Surgery Training and Research Hospital (approval number: 2020/57, dated August 18, 2020).

\section{Data collection}

The demographic data, baseline cardiovascular risk factors, clinical features, and laboratory values were obtained from patient files and hospital records. Routine blood tests were performed to assess complete blood count, liver and kidney functions, and lipid profile. Regarding follow-up parameters, major adverse cardiovascular and cerebrovascular events (MACCEs) were recorded. In addition, anatomical-based SYNTAX Score and novel SYNTAX Score II were recorded using the online calculator (www.syntaxscore.com) ${ }^{9}$.

\section{Assessment of coronary angiograms}

All angiograms and interventions were performed by experienced operators using standard methods and through either femoral or radial access. The left internal mammary artery (LIMA) and each aortic anastomosis were selectively injected. An aortic root angiogram was performed if the status of the SVG could not be determined by graft or stump injection. All angiograms were interpreted by the consensus of two interventional cardiologists blinded to the patients' clinical and laboratory data. A graft was described as failed if it had $70 \%$ or more stenosis or was completely occluded. If a graft had less than $70 \%$ stenosis and the whole course of the graft was visualized, it was described as patent. In sequential vein grafts, each segment was analyzed as a separate graft. Intraobserver and interobserver coefficients of variation [standard deviation (SD) of the differences between two observations divided by the mean value and expressed as a percent] were found to be $1.1 \%$ and $1.9 \%$, respectively.

\section{Statistical analysis}

Data were analyzed with the NCSS (Number Cruncher Statistical System) 2007 statistical software (Utah, USA) pocket program. In this study, data are expressed as mean $\pm S D$ for continuous variables and as counts and percentages for categorical variables. The Kolmogorov-Smirnov and Shapiro-Wilk tests were used to evaluate the distribution of continuous variables. The $\chi^{2}$ test and Fisher's exact test were used to analyze categorical variables. The Student's $t$-test was used for continuous variables with normal distribution and the values were presented as mean $\pm S D$. Comparison of intergroup continuous variables without normal distribution was analyzed using Mann-Whitney $U$-test. A $\mathrm{p}<0.05$ was considered to indicate statistical significance. Logistic regression analysis was used to assess the predictors of late graft failure. Variables with $p<0.05$ by univariate analysis were included in the multivariate logistic regression analysis model and the respective odds ratios (OR) with $95 \%$ confidence intervals $(\mathrm{CI})$ were calculated. All statistical tests were two-sided, and the level of significance was set at $\mathrm{p}<0.05$.

\section{RESULTS}

Of the 1,875 patients initially screened, a total of 280 patients were included in the study. Based on our data, 136 patients had at least one late SVG failure (study group), while 144 patients had patent SVG (control group). The patient characteristics are 


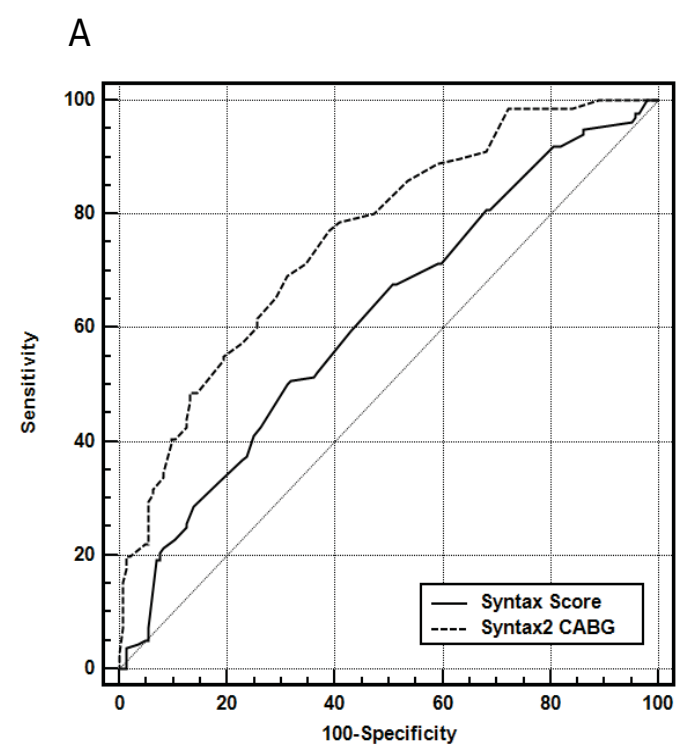

Receiver operating characteristic analysis for predicting late saphenous vein graft failure

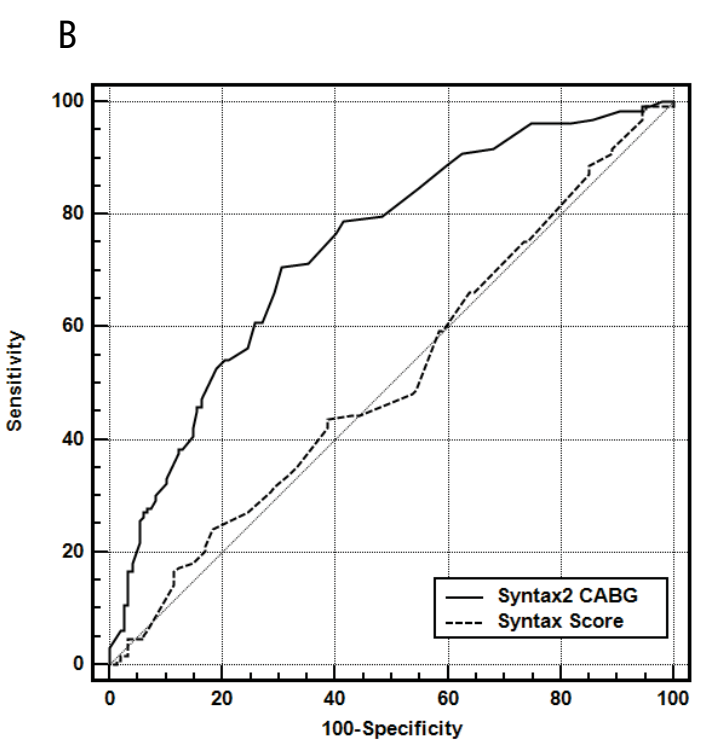

Receiver operating characteristic analysis for predicting major adverse cardiovascular and cerebrovascular events

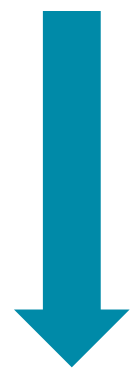

\section{1 patients were excluded from the study}

102 patients who underwent coronary angiography during the first year following CABG

239 patients who underwent emergent CABG

112 patients who underwent concomitant valve surgery 98 patients with a prior history of severe organ dysfunction 39 patients with a prior history of congenital heart disease 91 patients with a prior history of malignancy

Initially 1,194 patients were enrolled

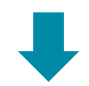

451 patients with late SVG failure (+)

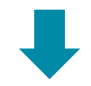

\section{5 patients excluded from the study}

164 patients failed to appear for more than 1 visit 45 patients excluded due to inadequate hospital recordings 106 patients excluded due to treatment discontinuation

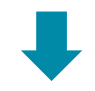

\section{6 patients (SVG failure (+) group)}

\section{3 patients with late SVG failure (-)}

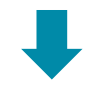

\section{9 patients excluded from the study}

309 patients failed to appear for more than 1 visit 98 patients excluded due to inadequate hospital recordings

192 patients excluded due to treatment discontinuation

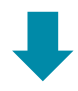

144 patients (SVG failure (-) group)

CABG: coronary artery bypass grafting; SVG: saphenous vein graft.

Figure 1. 1,875 patients undergoing coronary artery bypass grafting with a diagnosis of multivessel coronary artyery disease. 
summarized in Table 1. Both groups were similar in terms of demographic and clinical characteristics. On the other hand, history of hypertension (HT) and diabetes mellitus (DM) was significantly higher in patients with late SVG failure (+) compared to patients with late SVG failure (-), (96.32 versus $73.61 \%$; 61.76 versus $44.44 \%, \mathrm{p}<0.05)$. Baseline laboratory values were also compared between the two groups. However, serum uric acid level $(5.87 \pm 1.98 \mathrm{mg} / \mathrm{dL}$ versus $5.02 \pm 1.52 \mathrm{mg} / \mathrm{dL}, \mathrm{p}<0.05)$, neutrophil count $\left(5.54 \pm 1.39 \times 10^{3} / \mathrm{mm}^{3}\right.$ versus $4.90 \pm 1.33 \times 10^{3} / \mathrm{mm}^{3}$, $\mathrm{p}<0.05)$, and creatinine level $(1.05 \pm 0.36 \mathrm{mg} / \mathrm{dL}$ versus
$0.91 \pm 0.23 \mathrm{mg} / \mathrm{dL}, \mathrm{p}<0.05)$ were significantly higher in patients with late SVGs failure (-) compared to patients with late SVG failure (-). In addition, neutrophil-to-lymphocyte ratio was significantly higher in patients with late SVG failure $(+)$ compared to patients with late SVG failure (-) (2.92 \pm 1.38 versus $2.40 \pm 1.23, \mathrm{p}<0.05)$. Regarding echocardiographic measurements, patients with late SVG failure (+) had a lower estimated preprocedural left ventricular ejection fraction (LVEF) compared to those with late SVG failure (-), (47.74 \pm 11.17 versus $54.9 \pm 8.55, \mathrm{p}<0.05)$.

Table 1. Baseline characteristics of patients compared between groups.

\begin{tabular}{|c|c|c|c|c|}
\hline \multirow{2}{*}{\multicolumn{2}{|c|}{ Age }} & SVG Failure (-) n:144 & SVG Failure (+) n:136 & $p$ \\
\hline & & $58.52 \pm 8.1$ & $60.24 \pm 7.96$ & 0.074 \\
\hline \multirow{2}{*}{ Sex n (\%) } & Female & $25(17.36)$ & $35(25.74)$ & \multirow{2}{*}{0.088} \\
\hline & Male & $119(82.64)$ & $101(74.26)$ & \\
\hline \multicolumn{2}{|c|}{ Smoking history n (\%) } & 74 (51.39) & $85(62.50)$ & 0.061 \\
\hline \multicolumn{2}{|l|}{ DM (n/\%) } & $64(44.44)$ & $84(61.76)$ & 0.004 \\
\hline \multicolumn{2}{|l|}{ HT n (\%) } & $106(73.61)$ & $131(96.32)$ & 0.0001 \\
\hline \multicolumn{2}{|c|}{ Previous MI n (\%) } & $71(49.31)$ & $69(50.74)$ & 0.881 \\
\hline \multicolumn{2}{|c|}{ Previous PCI n (\%) } & $64(44.44)$ & $56(41.18)$ & 0.581 \\
\hline \multicolumn{2}{|c|}{ Syntax II score } & $25.52 \pm 9.62$ & $36.00 \pm 10.83$ & 0.0001 \\
\hline \multicolumn{2}{|c|}{ Syntax score } & $27.89 \pm 5.74$ & $30.17 \pm 5.91$ & 0.001 \\
\hline \multicolumn{2}{|l|}{ LVEF (\%) } & $54.9 \pm 8.55$ & $47.74 \pm 11.17$ & 0.0001 \\
\hline \multicolumn{2}{|c|}{ Total cholesterol (mg/dL) } & $201.06 \pm 52.92$ & $207.49 \pm 65.26$ & 0.366 \\
\hline \multicolumn{2}{|c|}{$\mathrm{HDL}(\mathrm{mg} / \mathrm{dL})$} & $39.63 \pm 12.92$ & $38.26 \pm 10.32$ & 0.330 \\
\hline \multicolumn{2}{|c|}{$\mathrm{LDL}(\mathrm{mg} / \mathrm{dL})$} & $130.55 \pm 42.48$ & $131.54 \pm 42.42$ & 0.846 \\
\hline \multicolumn{2}{|c|}{ Triglyceride (mg/dL) } & $192.87 \pm 91.17$ & $209.61 \pm 115.35$ & 0.178 \\
\hline \multicolumn{2}{|c|}{ Glucose (mg/dL) } & $137.17 \pm 56.44$ & $143.07 \pm 64.25$ & 0.415 \\
\hline \multicolumn{2}{|l|}{$\mathrm{HbA} 1 \mathrm{c}(\%)$} & $6.8 \pm 1.68$ & $7.18 \pm 2.08$ & 0.099 \\
\hline \multicolumn{2}{|c|}{ Uric acid (mg/dL) } & $5.02 \pm 1.52$ & $5.87 \pm 1.98$ & 0.003 \\
\hline \multicolumn{2}{|c|}{ Creatinine (mg/dL) } & $0.91 \pm 0.23$ & $1.05 \pm 0.36$ & 0.0001 \\
\hline \multicolumn{2}{|c|}{ eGFR (CKD-EPI) } & $99.73 \pm 23.46$ & $82.57 \pm 27.71$ & 0.0001 \\
\hline \multicolumn{2}{|c|}{ WBC $\left(10^{3} / \mathrm{mm}^{3}\right)$} & $9.03 \pm 2.69$ & $8.79 \pm 2.64$ & 0.452 \\
\hline \multicolumn{2}{|c|}{ Hemoglobin (g/dL) } & $13.58 \pm 1.60$ & $13.66 \pm 1.74$ & 0.701 \\
\hline \multicolumn{2}{|c|}{ Platelet $\left(10^{3} / \mathrm{mm}^{3}\right)$} & $242.97 \pm 68.21$ & $257.34 \pm 66.47$ & 0.079 \\
\hline \multicolumn{2}{|c|}{ Neutrophil $\left(10^{3} / \mathrm{mm}^{3}\right)$} & $4.90 \pm 1.33$ & $5.54 \pm 1.39$ & 0.0001 \\
\hline \multicolumn{2}{|c|}{ Lymphocyte $\left(10^{3} / \mathrm{mm}^{3}\right)$} & $2.26 \pm 0.65$ & $2.25 \pm 1.74$ & 0.966 \\
\hline \multicolumn{2}{|l|}{ NLR } & $2.40 \pm 1.23$ & $2.92 \pm 1.38$ & 0.001 \\
\hline \multicolumn{2}{|c|}{ Number of grafts } & $3.19 \pm 0.8$ & $3.35 \pm 0.8$ & 0.101 \\
\hline
\end{tabular}

SVG: saphenous vein graft; DM: diabetes mellitus; HT: hypertension; MI: myocardial infarction; PCI: percutaneous coronary intervention; LVEF: left ventricular ejection fraction; HDL: high-density lipoprotein; LDL: low-density lipoprotein; eGFR: estimated glomerular filtration rate; CKD: chronic kidney disease; EPI: chronic kidney disease epidemiology collaboration; WBC: white blood cell; NLR: neutrophil-to-lymphocyte ratio. 
Results of follow-up parameters are summarized in Table 2. The mean follow-up time for our study was 101.9 \pm 8.22 months. The mean length of intensive care unit stay and the mean length of hospital stay following surgery were compared between the two groups ( $p>0.05$ ). According to our data, incidence of nonfatal myocardial infarction and repeat revascularization were significantly higher in patients with late SVG failure $(+)$ compared to patients with late SVG failure (-) (38.24 versus $9.03 \%$; 41.18 versus $5.56 \%, \mathrm{p}<0.05)$. Of the whole cohort, MACCEs were significantly higher in patients with late SVG failure (+) compared to patients with late SVG failure (-) (66.91 versus $29.17 \%, \mathrm{p}<0.05)$.

Regarding assessment of coronary angiograms, both groups had higher rates of LIMA grafts (96.32 versus 95.14\%). According to our study, the rate of LAD-LIMA, DL-saphenous, IM-saphenous, CX-OM-saphenous, RCA-saphenous, RCA PDA SVG, and RCA PL SVG were compared between the two groups ( $p>0.05)$ (Table 2).

Table 2. Follow-up and angiographical details of patients compared between groups.

\begin{tabular}{|c|c|c|c|}
\hline & $\begin{array}{c}\text { Graft failure (-) } \\
n: 144\end{array}$ & $\begin{array}{c}\text { Graft failure (+) } \\
\text { n:136 }\end{array}$ & $p$ \\
\hline Mean ICU stay after CABG (day) & $1.09 \pm 0.49$ & $1.2 \pm 0.82$ & 0.345 \\
\hline Mean hospital stay after CABG (day) & $8.86 \pm 4.23$ & $8.99 \pm 4.82$ & 0.252 \\
\hline Mean follow up time (month) & $100.31 \pm 8.04$ & $103.49 \pm 8.41$ & 0.001 \\
\hline Postprocedural CVA n (\%) & $7(4.86)$ & $13(9.56)$ & 0.127 \\
\hline Postprocedural PAF n (\%) & 39 (27.08) & 45 (33.09) & 0.273 \\
\hline Peripheral intervention n (\%) & $11(7.64)$ & $16(11.76)$ & 0.242 \\
\hline Repeat revascularization n (\%) & $8(5.56)$ & $56(41.18)$ & 0.0001 \\
\hline Long-term CVA n (\%) & $20(13.89)$ & $26(19.12)$ & 0.238 \\
\hline Nonfatal MI n (\%) & $13(9.03)$ & $52(38.24)$ & 0.0001 \\
\hline MACCE (+) n (\%) & $42(29.17)$ & $91(66.91)$ & 0.0001 \\
\hline \multicolumn{4}{|c|}{ Assessment of coronary angiograms between groups } \\
\hline LAD LIMA graft n (\%) & $137(95.14)$ & $131(96.32)$ & 0.625 \\
\hline LAD SVG n (\%) & $9(6.25)$ & $16(11.76)$ & 0.106 \\
\hline LAD SVG failure $n(\%)$ & - & $4(2.94)$ & 0.001 \\
\hline D1 SVG n (\%) & $68(47.22)$ & $69(50.74)$ & 0.557 \\
\hline D1 SVG failure (n. \%) & - & $25(18.38)$ & 0.0001 \\
\hline IM SVG & $14(9.72)$ & $24(17.65)$ & 0.053 \\
\hline IM SVG failure n (\%) & - & $12(8.82)$ & 0.0001 \\
\hline LCX SVG n (\%) & $32(22.22)$ & $30(22.06)$ & 0.974 \\
\hline LCX SVG failure n (\%) & - & $10(7.35)$ & 0.001 \\
\hline LCX OM SVG n (\%) & $87(60.42)$ & $87(63.97)$ & 0.540 \\
\hline LCX OM SVG failure n (\%) & - & $44(32.35)$ & 0.0001 \\
\hline RCA SVG n (\%) & $71(49.31)$ & $68(50.00)$ & 0.908 \\
\hline RCA SVG failure n (\%) & - & $34(25.00)$ & 0.0001 \\
\hline RCA PDA SVG n (\%) & $40(27.78)$ & $30(22.06)$ & 0.269 \\
\hline RCA PDA SVG failure $n(\%)$ & - & $15(11.03)$ & 0.0001 \\
\hline RCA PL SVG n (\%) & $4(2.78)$ & $5(3.68)$ & 0.670 \\
\hline
\end{tabular}

ICU: intensive care unit; CABG: coronary artery bypass grafting; CVA: cerebrovascular accident; PAF: paroxysmal atrial fibrillation; MI: myocardial infarction; MACCE: major adverse cardiovascular and cerebrovascular events; LAD: left anterior descending; LIMA: left internal mammary artery; SVG: saphenous vein graft; D1: first diagonal branch; IM: intermediate artery; LCX: left circumflex artery; OM: optus marginalis; RCA: right coronary artery; PDA: posterior descending artery; PL: posterior lateral. 
Table 3. Logistic regression analysis of parameters for predictors of late saphenous venous graft failure

\begin{tabular}{|c|c|c|c|c|c|}
\hline \multirow{2}{*}{ Variables } & \multirow{2}{*}{ B } & \multirow{2}{*}{$p$} & \multirow{2}{*}{ OR } & \multicolumn{2}{|c|}{$95 \% \mathrm{Cl}$ for OR } \\
\hline & & & & Lower & Upper \\
\hline DM & 0.20 & 0.662 & 0.82 & 0.34 & 1.98 \\
\hline HT & 1.48 & 0.035 & 0.23 & 0.06 & 0.90 \\
\hline $\operatorname{MACCE}(+)$ & 0.65 & 0.292 & 1.91 & 0.57 & 6.36 \\
\hline Repeat revascularization & 1.92 & 0.01 & 0.15 & 0.03 & 0.63 \\
\hline Nonfatal MI & 1.34 & 0.068 & 0.26 & 0.06 & 1.11 \\
\hline $\operatorname{LVEF}(\%)$ & -0.04 & 0.065 & 0.96 & 0.92 & 1.00 \\
\hline Uric acid (mg/dL) & 0.16 & 0.248 & 1.17 & 0.90 & 1.54 \\
\hline Creatinine (mg/dL) & 0.13 & 0.899 & 0.88 & 0.13 & 6.22 \\
\hline eGFR (CKD-EPI) & -0.02 & 0.363 & 0.99 & 0.96 & 1.02 \\
\hline NLR & 0.14 & 0.504 & 1.15 & 0.76 & 1.73 \\
\hline Syntax II CABG score & 0.08 & 0.001 & 1.09 & 1.03 & 1.14 \\
\hline Syntax score & 0.01 & 0.897 & 1.01 & 0.93 & 1.08 \\
\hline
\end{tabular}

DM: diabetes mellitus; HT: hypertension; MACCE: major adverse cardiovascular and cerebrovascular events; MI: myocardial infarction; LVEF: left ventricular ejection fraction; eGFR: estimated glomerular filtration rate; CKD: chronic kidney disease; EPI: chronic kidney disease epidemiology collaboration; NLR: neutrophil-to-lymphocyte ratio; CABG: coronary artery bypass grafting.

The variables with $\mathrm{p}<0.05$ in univariate logistic regression analysis are listed in Table 3 and were included in multiple logistic regression analysis. After adjusting for confounding factors, prior history of HT and higher rates of repeat revascularization were found to be independent predictors of late SVG failure. In the receiver operating characteristic curve analysis, for anatomical SYNTAX Score, area under curve (AUC) was 0.617 for predicting late SVG failure (SE:0.033; 95\%CI 0.558-0.675) and was 0.761 for SYNTAX II CABG Score (SE:0.029; 95\%CI $0.706-0.810$ ). The predictive value of SYNTAX II CABG Score was found to be significantly higher than anatomical SYNTAX Score $(\mathrm{p}=0.001)$. Moreover, for anatomical SYNTAX Score, AUC was 0.514 for predicting MACCE (SE:0.035; 95\%CI 0.454-0.574) and was 0.741 for SYNTAX II CABG Score (SE:0.030; 95\%CI 0.685-0.791). The predictive value of SYNTAX II CABG Score was found to be significantly higher than anatomical SYNTAX Score $(\mathrm{p}=0.001)$ (Table 4).

\section{DISCUSSION}

In the present study, we investigated demographic and clinical features of patients undergoing isolated CABG and evaluated the factors associated with late SVG failure. Our results indicate that Syntax II Score could be a useful predictor for late SVG failure. To our knowledge, this is the first study
Table 4. Receiver operating characteristic analysis for predicting late saphenous vein graft failure and major adverse cardiovascular and cerebrovascular events.

\begin{tabular}{|c|c|c|c|c|}
\hline & AUC & SE & $95 \% \mathrm{Cl}$ & $p$ \\
\hline \multicolumn{5}{|l|}{ A } \\
\hline Syntax 2 CABG & 0.761 & 0.029 & $0.706-0.810$ & \multirow{2}{*}{0.001} \\
\hline Syntax Score & 0.617 & 0.033 & $0.558-0.675$ & \\
\hline \multicolumn{5}{|l|}{ B } \\
\hline Syntax 2 CABG & 0.741 & 0,030 & $0.685-0.791$ & \multirow{2}{*}{0.001} \\
\hline Syntax Score & 0.514 & 0,035 & $0.454-0.574$ & \\
\hline
\end{tabular}

AUC: area under curve; SE: standard error; CABG: coronary artery bypass grafting.

in literature to demonstrate the strong association between SYNTAX Score II and late SVG failure in patients undergoing isolated CABG.

CABG surgery is a well-established treatment modality for patients with multivessel CAD. On the other hand, SVG failure limits the long-term benefits of the procedure $^{10,11}$. Due to major adverse cardiac events associated with SVG failure, it is mandatory to maintain graft patency. Basically, SVG failure develops in three phases: early (less than 1 month), intermediate (1 month to 1 year), and late (beyond 1 year) ${ }^{12}$. Early SVG failure results from technical issues or thrombosis and usually occurs at the site of graft 
anastomosis. Factors associated with early SVG failure are endothelial injury, poor distal runoff, graft kinking, and small target vessel diameter ${ }^{12,13}$. The main pathological process that contributes to the development of intermediate SVG failure is progressive graft intimal hyperplasia. The underlying mechanism causing this pathologic condition is increased arterial pressure through the venous conduits. When saphenous veins are used as arterial conduits, alterations in hemodynamic status trigger intimal damage, fibrosis, platelet aggregation, release of growth factors, and smooth muscle cell proliferation. Progressive smooth muscle cell and fibroblast proliferation result not only in the development of neointimal hyperplasia but also in the luminal loss ${ }^{14-17}$.

On the contrary, late SVG failure develops as a result of an atherogenic process and is frequently observed over the damaged endothelium ${ }^{12}$. Previous studies showed that there were several atherosclerotic risk factors including age, race, gender, hypercholesterolemia, DM, HT, and chronic kidney disease associated with the development of late SVG failure ${ }^{18-20}$. In addition, histopathological studies investigating damaged SVG demonstrated the presence of necrotic core, calcification, and negative remodeling, which support the unfavorable effects of accelerated atherosclerosis ${ }^{21}$. According to human autopsy studies, SVG lesions older than 2 years were found to be more concentric and diffuse and more prone to rupture and occlude compared to native lesions ${ }^{22-24}$.

In our study, we observed a strong relationship between the prior history of HT, DM, reduced ejection fraction, CAD severity, and late SVG failure. With respect to assessing the severity of CAD, we used anatomical SYNTAX Score and novel SYNTAX II Score. This scoring system combined the clinical features (age, creatinine clearance, LVEF, left main CAD, sex, chronic obstructive pulmonary disease, and peripheral vascular disease) of patients along with anatomical characteristics of the coronary arteries (anatomical SYNTAX Score) replacing the previously used SYNTAX Score? ${ }^{9}$ Several studies confirmed this outcome, including Evaluation of the Xience Everolimus Eluting Stent versus Coronary Artery Bypass Surgery for Effectiveness of Left Main Revascularization trial that implemented this scoring system into the clinical practice and demonstrated more precise outcomes compared to former SYNTAX Score ${ }^{25}$. According to a meta-analysis conducted by Chen et al., SYNTAX Score II was superior to SYNTAX Score and played a substantial role in terms of predicting adverse clinical outcomes in patients who underwent coronary revascularization ${ }^{26}$. Outcomes of our study were compared with their result, which revealed
Syntax II score was superior to anatomical Syntax Score in terms of predicting SVG failure and MACCE ( $\mathrm{p}=0.001$, for both). Regarding real-world practice, the predictive value of SYNTAX Score II was also confirmed by an observational study conducted by Song et al., who analyzed the outcomes of 4,398 consecutive patients following three-vessel and/or unprotected LMCA-PCI by means of dividing their estimated SYNTAX II Scores into the tertiles (with cutoff points at 20 and 26). According to their study, mortality rate was significantly higher in the upper tertile compared to the intermediate or lower tertiles during the 2-year follow-up (2.7 versus $1.7 \%$ versus $0.5 \% ; \mathrm{p}<0.001)$. Multivariate analysis also showed that SYNTAX Score II was an independent predictor of 2-year mortality (hazard ratio, 1.66 [95\%CI 1.03-2.68]; $\mathrm{p}=0.04)^{27}$. However, in another study conducted by Li et al., there was no relationship between SVG failure and calculated SYNTAX II Scores ${ }^{28}$. Although their outcomes are inconsistent with our findings, their study had some limitations due to the nature of their study. According to their study, traditional risk factors, including HT, DM, and smoking, were not found related to SVG failure. Regarding the strong association between well-known atherosclerotic risk factors and higher SYNTAX Score II, it was inconceivable to achieve such an outcome. Therefore, claiming that there was no association between consensual traditional risk factors and SVG failure would create inconsistency and limit the generalizability of their results. According to a post hoc analysis of the Clopidogrel After Surgery for Coronary Artery Disease trial, the presence of HT, SVG diameter, grafting to the right coronary artery, and low quality of the target vessel correlate with the development of SVG hyperplasia or occlusion by 1 year after CABG. In addition, low target vessel quality and female sex were risk factors for SVG occlusion ${ }^{29}$. These parameters correlate with higher SYNTAX II Scores. They also demonstrated that the use of $\beta$-blockers and statins was associated with less SVG disease, confirming the importance of strict adherence to post-CABG medical treatment.

\section{Limitation}

The main limitation of the present study is that it was a single-center, retrospective experience with a relatively small sample size. Thus, further prospective studies with a larger population are needed to confirm our results. Although we investigated the predictors of late SVG failure, we did not investigate our patients with regard to occurrence of early and intermediate SVG graft failure. Due to exclusion of patients undergoing emergent CABG or concomitant mitral and aortic valve surgery, outcomes of this study cannot be applied to this population. 


\section{CONCLUSIONS}

Late SVG failure has diverse etiology and is associated with adverse clinical manifestations and often requires repeat revascularization. Despite various known risk factors, estimated SYNTAX Score II was found to be independent predictor of late SVG failure in patients undergoing isolated CABG. Due to its convenience and easy accessibility, this method can be applied to clinical routine.

\section{AUTHORS" CONTRIBUTIONS}

MD: Conceptualization, Data curation, Formal analysis, Methodology, Writing - original draft. OT: Conceptualization, Data curation, Formal analysis, Resources, Software, Writing - review \& editing. YA: Conceptualization, Formal analysis, Investigation, Methodology, Project administration, Software, Supervision, Validation, Visualization, Writing review \& editing.

\section{REFERENCES}

1. Lopes RD, Mehta RH, Hafley GE, Williams JB, Mack MJ, Peterson ED, et al. Relationship between vein graft failure and subsequent clinical outcomes after coronary artery bypass surgery. Circulation. 2012;125(6):749-56. https://doi. org/10.1161/CIRCULATIONAHA.111.040311

2. Gaudino M, Antoniades C, Benedetto U, Deb S, Di Franco A, Di Giammarco G, et al. Mechanisms, consequences, and prevention of coronary graft failure. Circulation. 2017;136(18):1749-64. https://doi.org/10.1161/CIRCULATIONAHA.117.027597

3. Ferguson Jr TB, Peterson ED, Coombs LP, Eiken MC, Carey ML, Grover $\mathrm{FL}$, et al. Use of continuous quality improvement to increase use of process measures in patients undergoing coronary artery bypass graft surgery: a randomized controlled trial. JAMA. 2003;290(1):49-56. https://doi.org/10.1001/jama.290.1.49

4. Holman WL, Sansom M, Kiefe $\mathrm{Cl}$, Peterson ED, Hubbard SG, Delong JF, et al. Alabama coronary artery bypass grafting project: results from phase II of a statewide quality improvement initiative. Ann Surg. 2004;239(1):99-109. https:// doi.org/10.1097/01.sla.0000103065.17661.8f

5. Björk VO, Ekeström S, Henze A, Ivert T, Landou C. Early and late patency of aortocoronary vein grafts. Scand J Thorac Cardiovasc Surg. 1981;15(1):11-21. https://doi. org/10.3109/14017438109101020

6. Roth JA, Cukingnan RA, Brown BG, Gocka E, Carey JS. Factors influencing patency of saphenous vein grafts. Ann Thorac Surg. 1979;28(2):176-183. https://doi.org/10.1016/s00034975(10)63777-0

7. Lichtenwalter $C$, de Lemos JA, Roesle M, Obel O, Holper EM, Haagen D, et al. Clinical presentation and angiographic characteristics of saphenous vein graft failure after stenting: insights from the SOS (stenting of saphenous vein grafts) trial. JACC Cardiovasc Interv. 2009;2(9):855-60. https://doi. org/10.1016/j.jcin.2009.06.014

8. Ong ATL, Serruys PW, Mohr FW, Morice MC, Kappetein AP, Holmes DR, et al. The SYNergy between percutaneous coronary intervention with TAXus and cardiac surgery (SYNTAX) study: design, rationale, and run-in phase. Am Heart J. 2006;151(6):1194-204. https://doi.org/10.1016/j. ahj.2005.07.017

9. Farooq V, van Klaveren D, Steyerberg EW, Meliga E, Vergouwe $Y$, Chieffo A, et al. Anatomical and clinical characteristics to guide decision making between coronary artery bypass surgery and percutaneous coronary intervention for individual patients: development and validation of SYNTAX score II.
Lancet. 2013;381(9867):639-50. https://doi.org/10.1016/ S0140-6736(13)60108-7

10. Yusuf S, Zucker D, Peduzzi P, Fisher LD, Takaro T, Kennedy JW, et al. Effect of coronary artery bypass graft surgery on survival: overview of 10-year results from randomised trials by the coronary artery bypass graft surgery trialists collaboration. Lancet. 1994;344(8922):563-70. https://doi.org/10.1016/ s0140-6736(94)91963-1

11. Davis KB, Chaitman B, Ryan T, Bittner V, Kennedy JW. Comparison of 15-year survival for men and women after initial medical or surgical treatment for coronary artery disease: a CASS registry study. Coronary Artery Surgery Study. J Am Coll Cardiol. 1995;25(5):1000-9. https://doi.org/10.1016/07351097(94)00518-u

12. Motwani JG, Topol EJ. Aortocoronary saphenous vein graft disease: pathogenesis, predisposition, and prevention. Circulation. 1998;97(9):916-31. https://doi.org/10.1161/01.cir.97.9.916

13. Sabik 3rd JF, Blackstone EH. Coronary artery bypass graft patency and competitive flow. J Am Coll Cardiol. 2008;51(2):126-8. https://doi.org/10.1016/j.jacc.2007.09.029

14. Nwasokwa ON. Coronary artery bypass graft disease. Ann Intern Med. 1995;123(7):528-45. https://doi.org/10.7326/00034819-123-7-199510010-00009

15. Schwartz SM, deBlois D, O'Brien ER. The intima. Soil for atherosclerosis and restenosis. Circ Res. 1995;77(3):445-65. https://doi.org/10.1161/01.res.77.3.445

16. Allaire $\mathrm{E}, \mathrm{Clowes} \mathrm{AW}$. Endothelial cell injury in cardiovascular surgery: the intimal hyperplastic response. Ann Thorac Surg. 1997;63(2):582-91. https://doi.org/10.1016/s00034975(96)01045-4

17. Zhang L, Peppel K, Brian L, Chien L, Freedman NJ. Vein graft neointimal hyperplasia is exacerbated by tumor necrosis factor receptor-1 signaling in graft-intrinsic cells. Arterioscler Thromb Vasc Biol. 2004;24(12):2277-83. https://doi.org/10.1161/01. ATV.0000147766.68987.0d

18. Hess CN, Lopes RD, Gibson CM, Hager R, Wojdyla DM, Englum BR, et al. Saphenous vein graft failure after coronary artery bypass surgery: insights from PREVENT IV. Circulation. 2014;130(17):1445-51. https://doi.org/10.1161/ CIRCULATIONAHA.113.008193

19. Efird JT, O'Neal WT, Gouge CA, Kindell LC, Kennedy WL, Bolin Jr P, et al. Implications of hemodialysis in patients undergoing coronary artery bypass grafting. Int J Cardiovasc Res. 2013;2(6):1000154. https://doi.org/10.4172/2324-8602.1000154. 
20. Yanagawa B, Algarni KD, Singh SK, Deb S, Vincent J, Elituv $R$, et al. Clinical, biochemical, and genetic predictors of coronary artery bypass graft failure. J Thorac Cardiovasc Surg. 2014;148(2):515-20.e2. https://doi.org/10.1016/j. jtcvs.2013.10.011

21. Sakakura K, Nakano M, Otsuka F, Yahagi K, Kutys R, Ladich $\mathrm{E}$, et al. Comparison of pathology of chronic total occlusion with and without coronary artery bypass graft. Eur Heart J. 2014;35(25):1683-93. https://doi.org/10.1093/eurheartj/ eht422

22. Yahagi K, Kolodgie FD, Otsuka F, Finn AV, Davis HR, Joner $M$, et al. Pathophysiology of native coronary, vein graft, and in-stent atherosclerosis. Nat Rev Cardiol. 2016;13(2):79-98. https://doi.org/10.1038/nrcardio.2015.164

23. Yazdani SK, Farb A, Nakano M, Vorpahl M, Ladich E, Finn AV, et al. Pathology of drug-eluting versus bare-metal stents in saphenous vein bypass graft lesions. JACC Cardiovasc Interv. 2012;5(6):666-74. https://doi.org/10.1016/j.jcin.2011.12.017

24. Muto A, Model L, Ziegler K, Eghbalieh SDD, Dardik A. Mechanisms of vein graft adaptation to the arterial circulation: insights into the neointimal algorithm and management strategies. Circ J. 2010;74(8):1501-12. https://doi.org/10.1253/ circj.cj-10-0495

25. Campos CM, van Klaveren D, Farooq V, Simonton CA, Kappetein AP, Sabik 3rd JF, et al. Long-term forecasting and comparison of mortality in the evaluation of the xience everolimus eluting stent vs. coronary artery bypass surgery for effectiveness of left main revascularization (EXCEL) trial: prospective validation of the SYNTAX score II. Eur Heart J. 2015;36(20):1231-41. https://doi.org/10.1093/eurheartj/ehu518

26. Chen JY, Tang B, Lin YQ, Ru Y, Wu MX, Wang X, et al. Validation of the ability of SYNTAX and clinical SYNTAX scores to predict adverse cardiovascular events after stent implantation: a systematic review and meta-analysis. Angiology. 2016;67(9):820-8. https://doi.org/10.1177/0003319715618803

27. Song Y, Gao Z, Tang X, Ma M, Jiang P, Xu J, et al. Usefulness of the SYNTAX score II to validate 2-year outcomes in patients with complex coronary artery disease undergoing percutaneous coronary intervention: a large single-center study. Catheter Cardiovasc Interv. 2018;92(1):40-7. https://doi.org/10.1002/ ccd. 27321

28. Li JH, Song $X T$, Yang $X Y$, Zhang $W Y$, Xing HR. Relevance of SYNTAX score for assessment of saphenous vein graft failure after coronary artery bypass grafting. Chronic Dis Transl Med. 2019;6(1):55-61. https://doi.org/10.1016/j.cdtm.2019.05.005

29. Une D, Kulik A, Voisine P, May ML, Ruel M. Correlates of saphenous vein graft hyperplasia and occlusion 1 year after coronary artery bypass grafting: analysis from the CASCADE randomized trial. Circulation. 2013;128(11 Suppl 1):S213-8. https://doi.org/10.1161/CIRCULATIONAHA.112.000328 